\title{
A concept for the integrated 3D flow, heat transfer and structural calculation of compact heat exchangers
}

\author{
F. Yang, K. Mohrlok, U. Schaeffer \& R. Stier \\ Department of Simulation and Testing, Modine Europe GmbH, Germany
}

\begin{abstract}
This article describes a concept and methodology for the modelling of compact heat exchangers with arbitrary flow arrangement. The new model enables integrated virtual testing including simulation of thermal and hydraulic performance, cavitation behavior, and thermal structural stresses. In order to limit the calculation cost, the problem solving is split into two steps: firstly the CFD turbulator characteristics will be determined with very fine geometric resolution for the heat transfer surface related micro physics; secondly the global CFD conjugate heat transfer will be calculated with a coarse mesh by use of the porous media concept. A key issue in the second step is the representation of the turbulator characteristics which are dependent on the arbitrary flow direction and the local Reynolds and Prandtl numbers. Unlike a usual porous media approach in which the turbulator solid is replaced, the global flow and heat transfer calculation is carried out here by use of the user-developed coding and the StarCCM+/StarCD model of conjugate heat transfer in anisotropic porous media. With the calculated temperature distribution in the structure used as loading, a thermal stress analysis can be performed to evaluate the thermo-mechanic fatigue life of heat exchangers. Measurement shows that the predicted heat transfer performance and the pressure losses with the described multi-scale calculation strategy compares well with the experimental data with a maximum deviation of about $10 \%$.

Keywords: liquid to liquid heat exchanger, multi scale calculation, turbulator properties in arbitrary flow direction, conjugate heat transfer in anisotropic porous media, thermal and hydraulic characteristics, thermal structural stresses, heat exchanger model, cavitation identification, CFD calculation, experimental validation.
\end{abstract}




\section{Introduction}

The performance determination of a compact heat exchanger is mainly carried out by experimental measurements. CFD calculation of its characteristics remains a challenging task, especially for layered oil coolers. Heat transfer surfaces in compact heat exchangers consist of fins or dimples as turbulators with normally periodical and very small geometrical features (see Figure 1). The resolution of these fine, structure-related flow physics requires very fine grids and tremendous computational efforts. This has made such a calculation for the industrial application almost impossible.

The 1D $\varepsilon$-NTU method for the determination of heat transfer performance $[1,2,6]$ uses the measured mean heat transfer data for turbulators. This only works for typical flow arrangements such as cross flow heat exchangers, e.g. a radiator in automotive applications. For a layered oil cooler, however, the specification of such a flow arrangement is not possible because of the $2 \mathrm{D} / 3 \mathrm{D}$ nature of the flow pattern of the oil and the coolant side. Further issues are bypasses and inhomogeneous inlet flows, which are also difficult to be taken into account.

Usual 3D CFD calculations for oil coolers (oil-to-coolant heat exchangers) use the following approach in order to reduce the requirements on calculation resources: it is often restricted to one fluid flow of either the oil or the coolant side, and is normally based on the porous media concept so that the heat transfer is not considered.

The relatively advanced concept of effective heat conductivity, in which the turbulators and fluid are replaced by a new fluid with higher heat conductivity, could theoretically capture heat transfer within a CFD calculation. Unfortunately this does not work well due to the different nature between convective heat transfer and heat conduction, aside from the complicated determination of such effective heat conductivity coefficients. An additional essential drawback of the concept is that the temperature distribution in the fins cannot be obtained, which is necessary for thermal structural stress analysis.
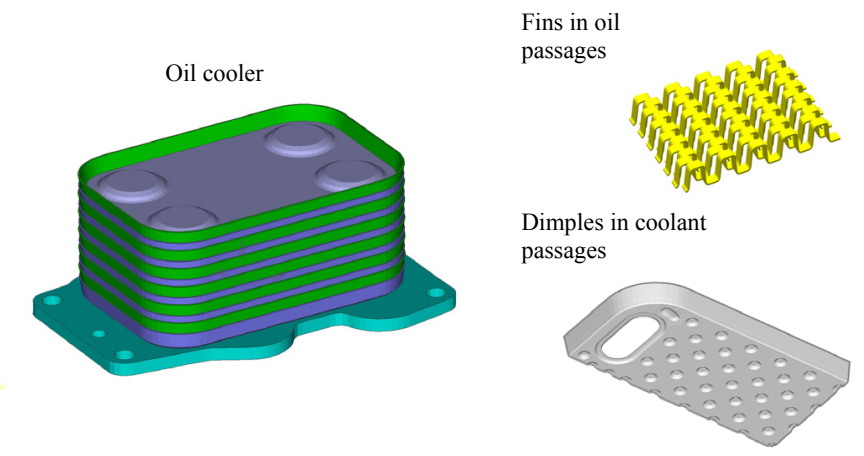

Figure 1: Layered oil cooler and the features of their heat transfer surfaces. 


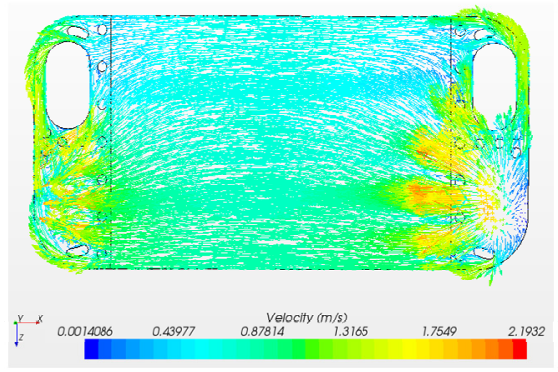

a)

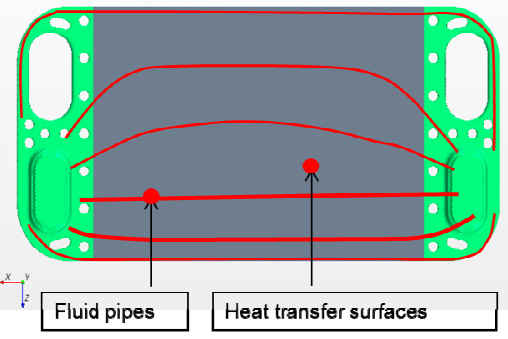

b)

Figure 2: 1D-3D mix model for the conjugate heat transfer calculation: a) real fluid velocity distribution; b) 1D model for the fluid flow and 3D model for the solid.

A further sophisticated approach for the conjugate heat transfer is to use the 1D-3D mix model as shown in Figure 2, which is often applied in connection with a FE thermal structural analysis. Because of the restriction of the calculation cost, the turbulators in the model are normally also not resolved, but modelled with coarser equivalent anisotropic heat conducting elements. However, the 1D-3D approach demands an accurate definition of the fluid pipes, the equivalent correspondent heat transfer surfaces, and the heat transfer coefficient distribution. For a cross or parallel flow heat exchanger [5] with one flow direction per fluid, this can be done with reasonable effort. But for the layered oil cooler it is almost impossible because of the arbitrary flow directions in the oil and coolant passages.

To overcome the difficulties mentioned above, a new calculation concept has been developed. This approach incorporates an integrated 3D flow, heat transfer and structural calculation of heat exchangers, and is especially applicable to layered oil coolers. With this new approach, not only the prediction of the hydraulic and heat transfer characteristics can be performed, but also the location of cavitation can be identified. In addition, a subsequent thermal structural stress analysis as well as an estimation of the thermal fatigue life can be carried out. Design optimizations such as bypass configuration, different inlet conditions and orientation changes of the fins can also be easily evaluated.

\section{Concepts and method of the integrated multi scale calculation}

The new concept of the heat transfer performance and thermal stress calculation is based on a multi scale strategy as shown in Figure 3 for a layered heat exchanger. It consists of two main steps:

The first step is the micro scale calculation. The micro scale calculation includes the following: the CFD determination of the fin properties related to the micro physics, such as hydraulic and heat transfer characteristics of the fins 


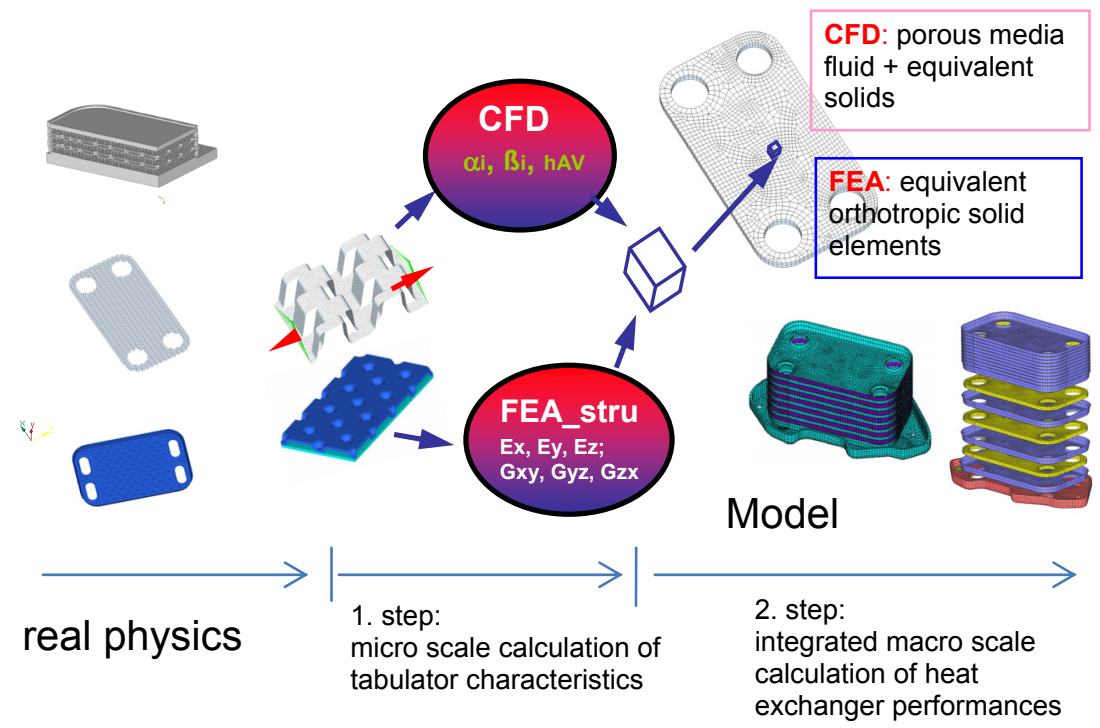

Figure 3: New concept for the heat transfer and stress calculation.

depending on local flow orientation, Reynolds number and Prandtl number; and the FE determination of the equivalent orthotropic fin stiffness for structural elements.

The second step is the macro scale calculation, which includes the global CFD and the global FE structural calculation. The global CFD calculation is performed by using the results of the micro scale calculation and the concept of conjugate heat transfer in anisotropic porous media. The global FE thermo structural stress analysis is carried out by modelling the fins with orthotropic structural elements and using the temperature distribution from the global CFD calculation as loads.

Unlike the usual porous media model, the anisotropic porous media concept for conjugate heat transfer models each small portion of space in the finned passages with both a porous fluid and a porous solid cell to represent this part of the heat exchanger. In this model the solid cell has the equivalent anisotropic heat conductivities of the fins while the fluid cell has the local pressure losses characteristics, which have been determined in the first step of the strategy. The heat transfer between the fluid and solid cell are modelled as source and sink term in the energy equation of the media, respectively, based on the micro scale calculation.

With this concept, the macro scale calculation of fluid flow, heat transfer and the structural stresses are not based on a fine grid, which can resolve micro physics related to the geometrical features of the heat transfer surfaces, but a much coarser grid, which needs only to fulfil the requirements to represent the local averaged performance characteristics. This reduces the needed calculation 
resources and cost to a small percentage of the original calculation task and allows virtual testing of compact heat exchangers with typical or non-typical flow arrangements on a workstation.

\section{Generation of the turbulator characteristics of flow and heat transfer}

Although the calculation of the fins (turbulators) in the micro scale is a normal CFD or FE task, the effective generation of the characteristics, which is flow direction dependent, needs some special considerations because of the boundary conditions.

\subsection{CFD fin properties calculation}

Unlike in a radiator or an air-cooled charge air cooler, in a finned passage of an oil cooler, the thermodynamic state varies while the flow direction and magnitude change with location.

If the calculation domain boundaries are chosen parallel or normal to the flow direction, the domain which represents a minimal periodic flow pattern will change its orientation and size with flow direction. In this case the boundary conditions are cyclic on the side parallel to the flow direction and partially cyclic on the other two sides. However, many calculation domains with different sizes must be used to calculate the flow direction dependent fin characteristics.

An effective way to carry out the CFD calculation of the fin properties [3] is delineated in Figure 4. In this case only one calculation domain is needed for all the calculations, which is chosen as a geometrically minimal periodical segment. The CFD domain used includes the fluid volume, fins and tube solids. The flow is treated therefore as hydraulic and thermal fully developed in two directions,
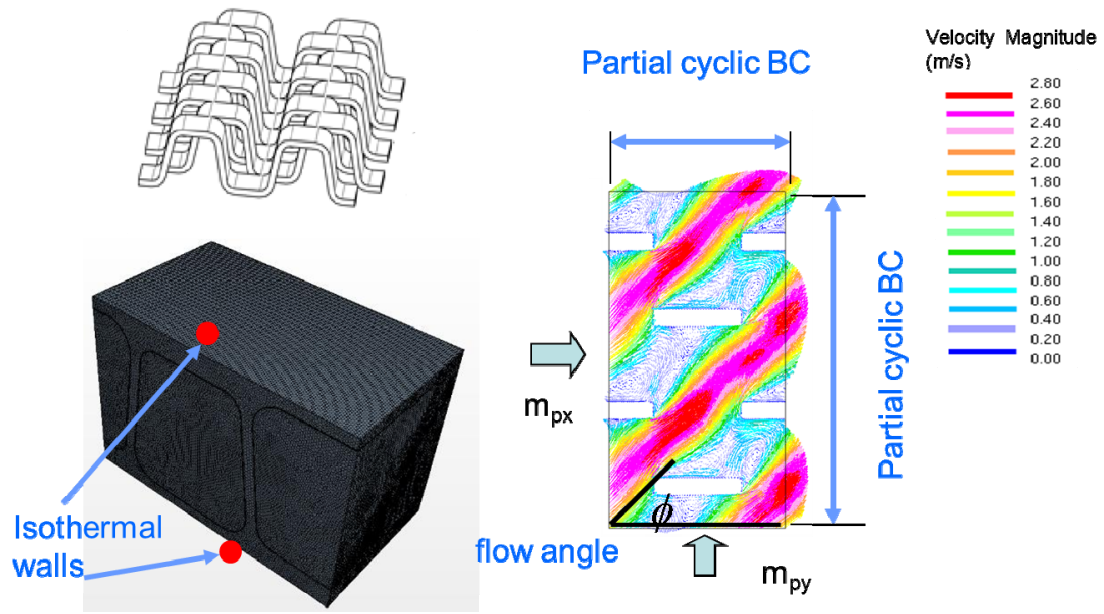

Figure 4: Flow domain and boundary conditions for the fin calculation. 
i.e. there are two sets of partial cyclic boundary conditions. This enables an effective calculation of fin characteristics for different flow velocity magnitude and directions using the same flow domain and mesh, but with different mass flow rates.

\subsection{Measurements of the fin characteristics}

In order to correlate the micro scale CFD results and to capture deviations from CAD geometry to manufactured and brazed samples, measurements have been performed.

Figure 5 shows the experimental platform for the fin characteristic measurements. The sample is a mini cross flow heat exchanger with the same fins and fin orientation to the flow for both cold and hot stream sides.

With different fluids, inlet temperature, mass flow rate and fin orientation the dimensionless fin characteristics can be obtained by measurement and regression. Caution must be taken here for flow angles other than $0^{\circ}$ and $90^{\circ}$ because the fluid is inclined to flow in the fin direction if the samples are not long enough in the designated flow direction, which will cause false measurement results (compare streamlines from CFD in Figure 6).

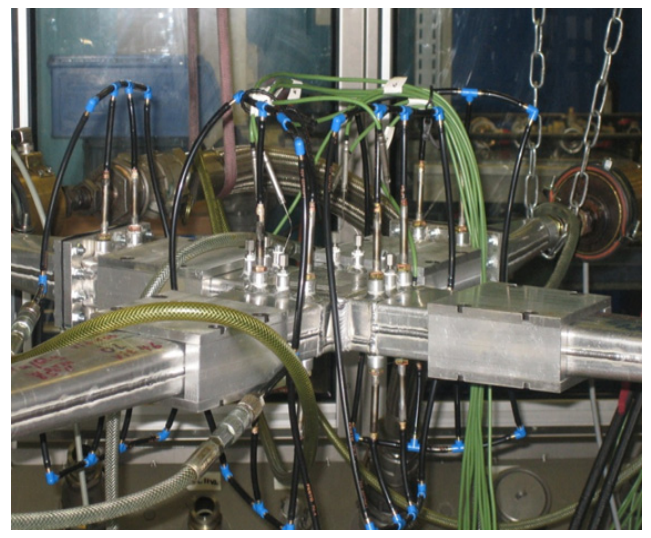

Figure 5: Experimental configuration for the fin measurements.

\subsection{Representation of the fin characteristics}

The flow through the turbulators such as fins or a dimple pattern could be in the laminar, transition or turbulent regimes. For the use in the macro scale calculation, the fin properties, i.e. pressure losses coefficient $(\xi)$ and Nusselt number $(\mathrm{Nu})$, for a fixed flow angle can be represented as continuously piecewise smooth functions of the Reynolds number (Re) and the Prandtl number (Pr):

$$
\xi=C o \cdot R e^{m 0} ; N u=C \cdot R e^{m 1} \cdot \operatorname{Pr}^{m 2}
$$

where $\mathrm{C}_{0}, \mathrm{C}, \mathrm{m}_{0}, \mathrm{~m}_{1}$ and $\mathrm{m}_{2}$ are coefficients and exponents depending on flow regime and flow direction. 
Figure 6 shows a comparison of the calculated and measured fin properties for a fixed Reynolds as a function of flow angle. To better illustrate the fin properties' dependence on flow angle, $\phi$, a polar coordinate system is used. Besides the good agreement with the measurements, it can also be noted that the calculated data fit well to the following formula:

$$
\zeta^{\phi}=\zeta_{0} \cos ^{2} \phi+\zeta_{90} \sin ^{2} \phi \quad \mathrm{Nu}_{\phi}=\mathrm{Nu}_{0} \cos ^{2} \phi+\mathrm{Nu}_{90} \sin ^{2} \phi
$$

These equations are derived from a porous media consideration using only fin characteristics at two flow angles, $0^{\circ}$ and $90^{\circ}$. This can be very useful if the fin properties' data are only available in these two directions.

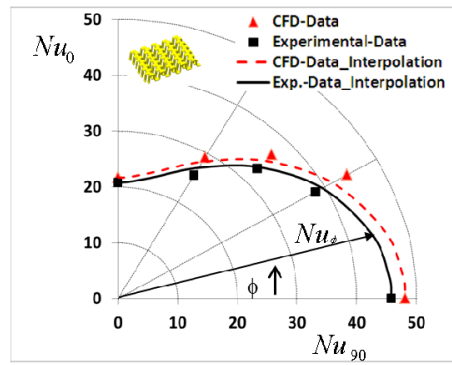

a)

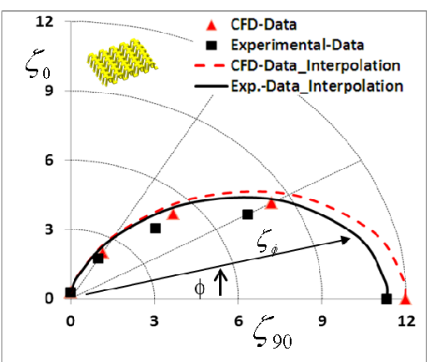

b)

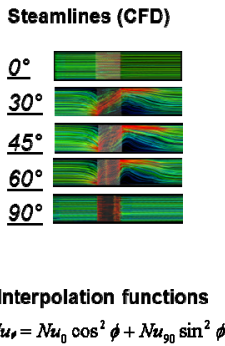

$\zeta_{\phi}=\zeta_{0} \cos ^{2} \phi+\zeta_{00} \sin ^{2} \phi$

$\mathrm{Nu}, \zeta$ against $\phi$ for $R \mathrm{e}=1000$

Figure 6: Fin properties over flow angles in polar coordinate system: a) Nusselt number; b) pressure losses coefficient.

\section{Calculation of the hydraulic and thermal performance of a compact heat exchanger}

If the fin characteristics are obtained as above described, the macro scale calculation with coarser mesh can be performed. The macro scale calculation does not need the resolution of the fins and the fin heat transfer surface related micro physics that the anisotropic porous media model with conjugate heat transfer required.

\subsection{Implementation of the concept for the CFD heat exchanger calculation}

For the calculation of the global flow fields as well as the hydraulics and thermal performance the commercial CFD code StarCD/StarCCM+ $[7,8]$ is used.

The implementation of the calculation concept described in section 2 is schematically shown in Figure 7. Two sets of user coding are used. One set is for the fluid properties, which are dependent on the thermodynamic state variables from the CFD flow field solution. The other is for the porous media parameters, i.e. the turbulator characteristics, which form the main embodiment of the work $[4,9]$. The turbulator characteristics for an arbitrary flow angle are calculated in the user coding by spline interpolation of the values of five known flow angles. 


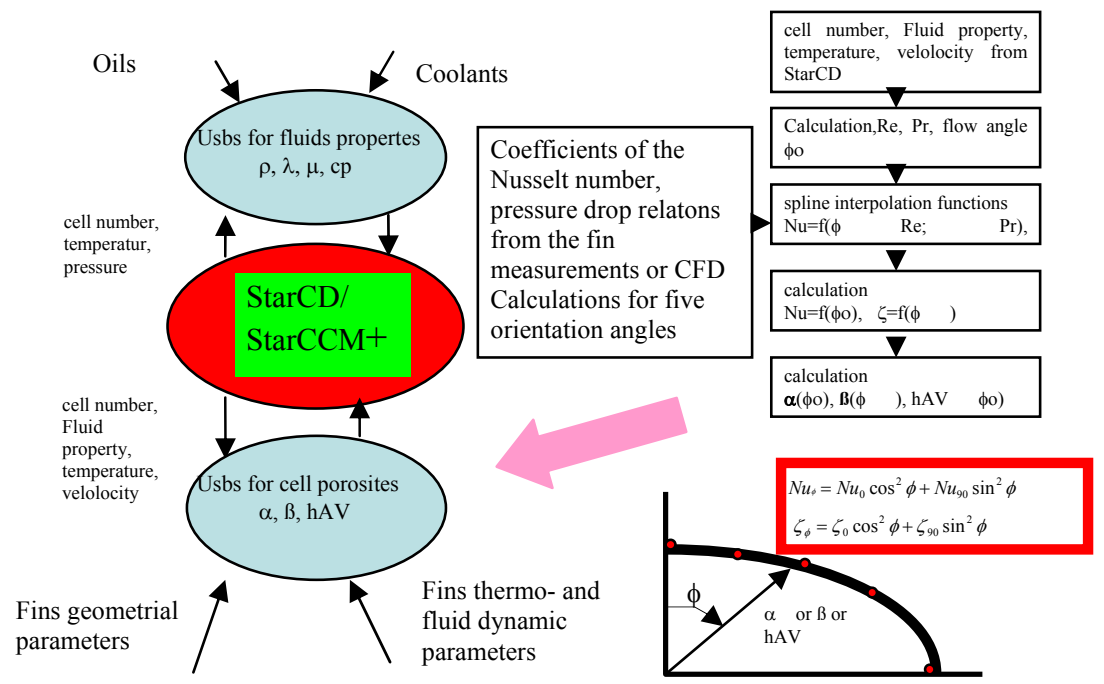

Figure 7: Flow chart of the calculation algorithms.

For the case in which only the values by $0^{\circ}$ and $90^{\circ}$ are known, the analytical interpolation formulas mentioned above can be applied.

\subsection{CFD oil-to-coolant heat exchanger calculation}

Using the procedure given above, a calculation for an oil cooler [4] is carried out. The oil cooler is finned both in the oil passages and in the coolant passages. For manufacturing reasons there are bypasses around the fin blocks and around the oil and coolant ports. The mesh for the calculation is generated automatically in Star CCM+. Although the geometry is very complicated, using the new features of the code, the calculation for one operating point can be finished very quickly.

Figure 8 shows the calculated global temperature distributions of the solid of the coolant and of the oil body. The two outer coolant passages are colder than the interior passages because of the arrangement of the oil and coolant passages. The effect of the bypasses in the oil and coolant passages can also be easily identified.

The temperature distributions in the section through the oil ports are shown in Figure 9. A clear image of the temperature gradients both in the flow direction and in the passage height direction can be obtained for the oil and the coolant side. The temperature distributions in the fins (porous solid), which are also the load for the structural stress analysis, beside the temperature of the tube plates (real solid), reflect the fin efficiencies. 


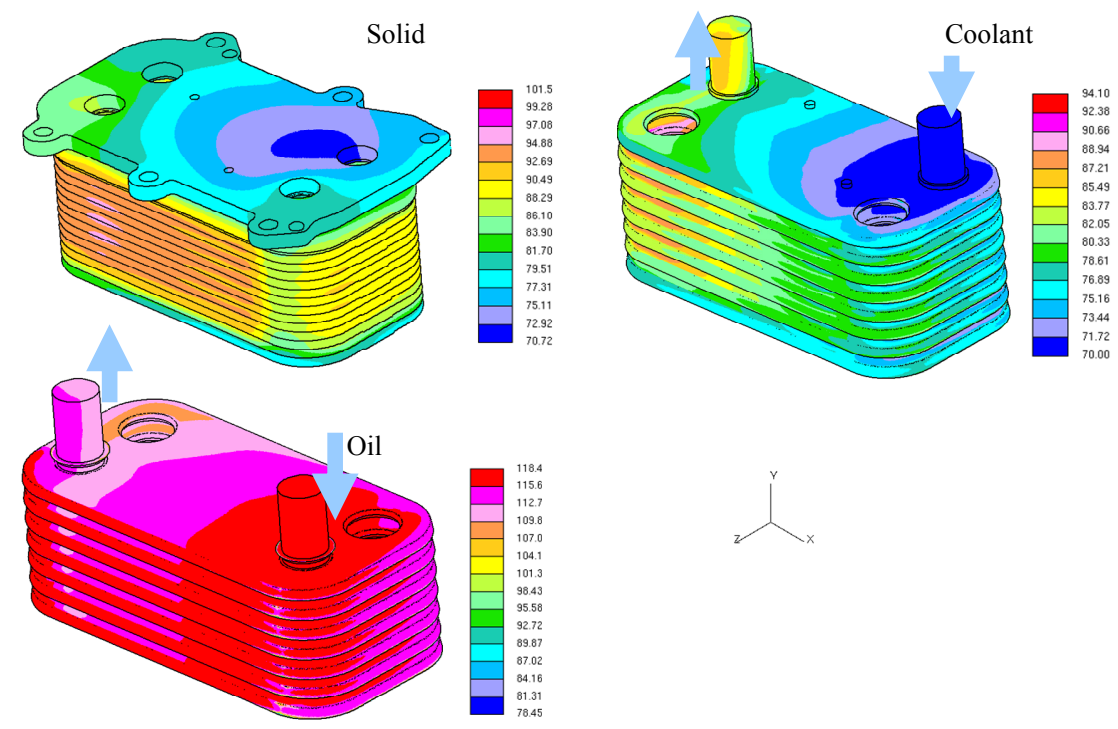

Figure 8: Global temperature distributions from $\operatorname{CFD}\left({ }^{\circ} \mathrm{C}\right)$.

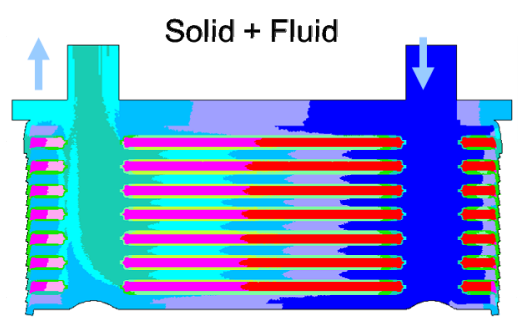

a)

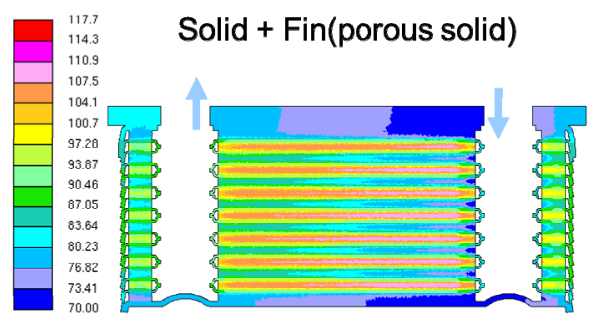

b)

Figure 9: Temperature distributions in the section through the oil ports $\left({ }^{\circ} \mathrm{C}\right)$, a) in solids and fluids; b) in solids and fins (porous solid).

\subsection{Measurements of characteristics of an oil cooler}

Figure 10 shows the test set up for oil coolers where the measurements for the pressure drop in both fluids and the heat transfer are carried out. The instrumentation utilized enables a measurement uncertainty of less than $5 \%$. 


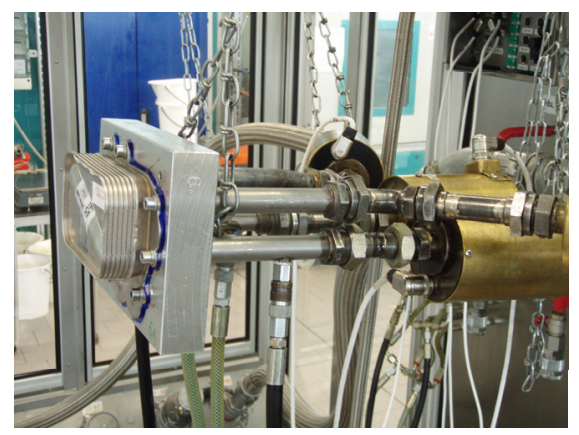

Figure 10: $\quad$ Test set up for oil coolers.

\subsection{Comparison between the calculated and measured heat exchanger characteristics}

The characteristics of an oil cooler, calculated with StarCD4.16/StarCCM+7.06 are shown in Figure 11. The calculation covers $20 \%$ to $100 \%$ of the maximum mass flow rates of the measured oil and coolant, respectively. The results agree well with the measurements. The deviation between calculation and measurement is less than $10 \%$ for oil and coolant side pressure drop as well as for heat transfer performance.
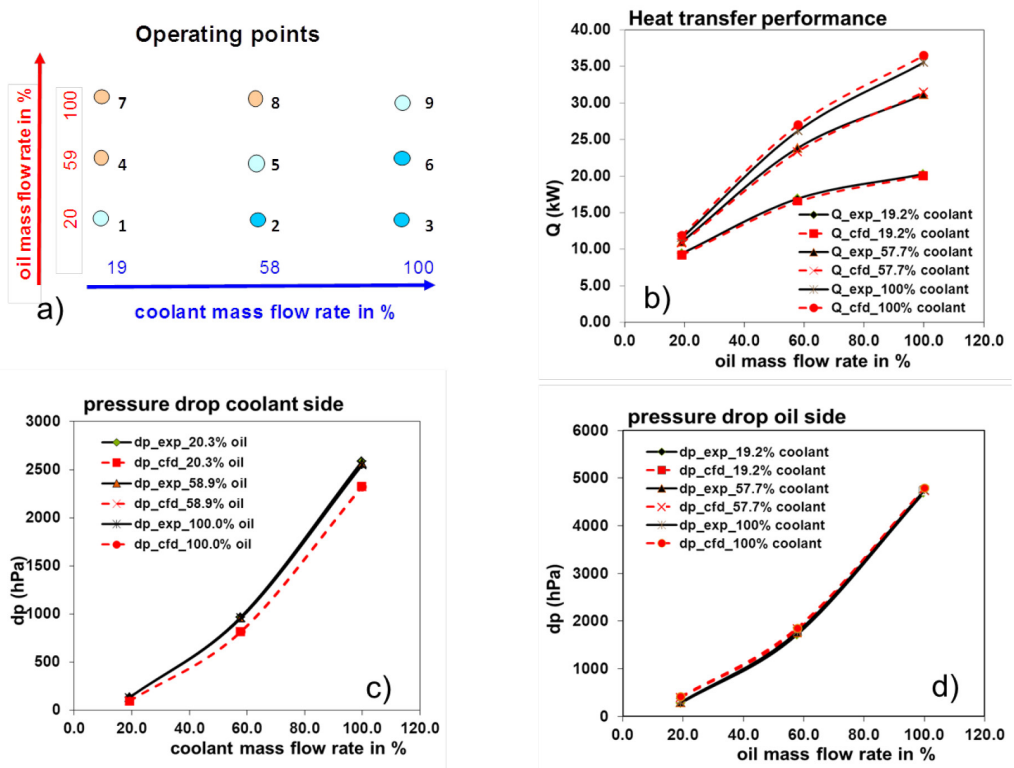

Figure 11: Comparison between CFD calculated and measured heat exchanger characteristics: a) operating points; b) heat transfer performances; c) pressure drop coolant side; d) pressure drop oil side. 


\subsection{Cavitation identification}

In some operating conditions not only thermal or other mechanical stresses could cause damage to a liquid-to-liquid heat exchanger but also cavitation does.

The cavitation potential of the flow is dependent on the local static pressure and the local fluid temperature which determine the fluid saturation pressure. It can be expressed as the cavitation number $(\mathrm{Ca})$ :

$$
C a=\frac{p-p_{v}}{\frac{1}{2} p V^{2}}
$$

where $p$ and $p_{v}$ are the local static and saturation pressure respectively, $\rho$ is the density of the fluid and $\mathrm{V}$ is the local velocity.

With the CFD-calculated quantities the cavitation number distributions can be obtained as shown in Figure 12. For regions in the figure with $\mathrm{Ca}<0$, cavitation damage is expected.

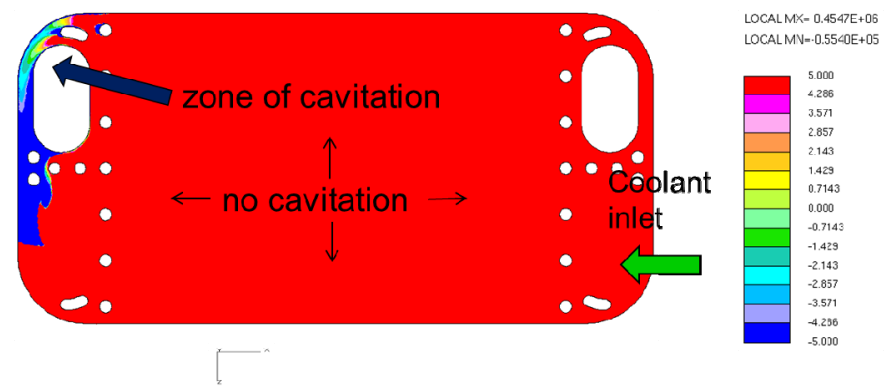

Figure 12: Cavitation number distributions.

\section{Thermal structural stress analysis}

The thermal structural stress analysis of the oil cooler is carried out with ANSYS V12.

As mentioned above, the macro-scale calculation does not resolve geometrical features of the fins. The fins in the FE structural model are represented with the so-called equivalent orthotropic structural elements. Their anisotropic Young's modulus and shear modulus are determined with a unit load and the smallest periodic segment of the fins.

The temperature load obtained from the macro scale CFD analysis can be mapped onto a FEA model to perform a global structural stress analysis. Figure 13 shows the calculated stress distribution in the structure.

If there is interest in the determination of the real fin stresses, a locally detailed mesh of the real fin or the sub-model technique should be used. As for determining the loads of the structure, the global CFD temperature distribution can also be mapped onto this portion of the fully resolved fins. 


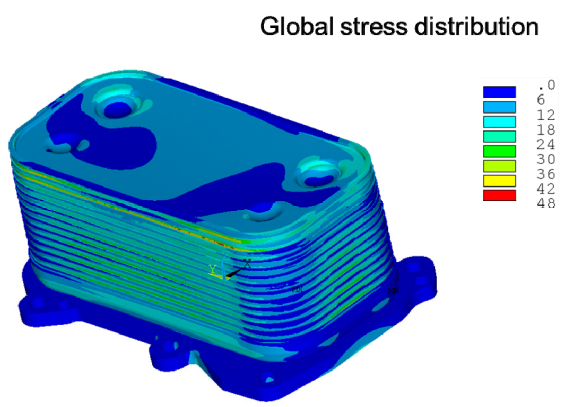

Figure 13: The calculated structural stresses of the oil cooler (MPa).

\section{Conclusions}

The presented concept allows for an effective multi-physics simulation of all types of compact heat exchangers with typical or non-typical flow arrangement. Heat exchangers that can be modelled include oil coolers, charge air coolers, exhaust gas recirculation coolers and radiators.

The procedure takes all local operating conditions into account which have an impact on heat transfer and pressure drop. It delivers the required thermal and hydrodynamic characteristics of a compact heat exchanger with a maximum deviation of $10 \%$ in comparison to the measurements.

Besides cavitation identification in liquids, the procedure delivers required temperature distributions in conventional solid elements as well as orthotropic solid elements used for turbulators so that a FE thermal structural stress calculation for durability predictions of heat exchangers can be easily carried out.

Because of the calculation accuracy and low calculation cost, the procedure can serve as a design optimization tool on normal workstations.

\section{References}

[1] Yang F., Opferkuch F., CFD Optimization of Heat Exchangers with Regard to their Fine Structures, Proceedings of the 1st mode Frontier Users' Meeting in Germany, Stuttgart, Oct. 2007.

[2] Opferkuch F., Yang F. et al., New Dimensioning Concepts of Compact Heat Exchangers, Proceedings of the VTMS conference 8, Nottingham, May, 2007.

[3] Janwe S., Yang F., Flow and heat transfer analysis of offset fins for arbitrary flow directions. Modine Europe internal report, Filderstadt, Sept. 2011.

[4] Yang F., Thumm S., Integrated flow, heat transfer and thermo stresses calculation of an oil cooler, Modine Europe internal report, Filderstadt, Dec. 2011.

[5] Urquiza E., Transient Thermal, Hydraulic, and Mechanical Analysis of a Counter Flow Offset Strip Fin Intermediate Heat Exchanger using an 
Effective Porous Media Approach, PhD-dissertation of University of California Berkley, fall 2009.

[6] Modine Heat Management Software (HMS), Modine, 2011.

[7] StarCD V.4.16 Methodology, CD-Adapco, 2011.

[8] StarCCM+ V7.06 User manual, CD-Adapco, 2012.

[9] Hariprasath, G., Yang F., translation of the user coding from Fortran for StarCD to Java for StarCCM+, Modine global internal report, Filderstadt, Nov. 2013. 